\title{
Correction to: "Silent Hypoxemia" Leads to Vicious Cycle of Infection, Coagulopathy and Cytokine Storm in COVID-19: Can Prophylactic Oxygen Therapy Prevent It?
}

\author{
Anshika Chauhan $^{1} \cdot$ Rajandeep Kaur $^{1} \cdot$ Prantar Chakrabarti $^{2} \cdot$ Arnab Pal $^{1}$ (D)
}

Published online: 29 April 2021

(C) Association of Clinical Biochemists of India 2021

\section{Correction to: Ind J Clin Biochem}

https://doi.org/10.1007/s12291-021-00967-0

The original article was published incorrectly. The author name "Prantar Chakrabarti" was misspelled as "Prantar Chakrbarti".

The original article can be found online at https:// doi.org/10.1007/s12291-021-00967-0.

Arnab Pal

pal.arnab@pgimer.edu.in

1 Department of Biochemistry, Post Graduate Institute of Medical Education and Research, Chandigarh, India

2 Vivekananda Institute of Medical Sciences, Kolkata, India 